\title{
Psycholinguistic Tools for Decoding Suggestive Potential of Verbal Models
}

\author{
T. Rogozhnikova \\ Department of Language Communication and Psycholinguistics \\ Ufa State Aviation Technical University \\ Ufa, Russia \\ burzian@yandex.ru
}

\begin{abstract}
The article deals with the tools for decoding a suggestive potential of verbal models in different languages (Russian, English, German, Bashkir and Tatar). The suggestive potential is defined by the author as a "verbal model strength" that can be decoded and measured. Being correlated with inner form, the potential is realized through the mechanism of association. The author has defined eight tools which promote revealing the suggestive resources of any verbal model. The first tool deals with studying rhythmic activity of the brain while presenting verbal stimuli. The second one relates to the analysis of psychosemantics of color and associative color of models. The third one bases on possible analogies with the models used in quantum physics. The fourth one touches upon the rhythmic organization and rhythmic codes of the models. The fifth tool is oriented on the analysis of language phenomena in the context of different perception modalities. The sixth one focuses on ranking emotional-evaluative features characteristic of verbal model. Suggestive resources of musical discourse are analyzed by means of the seventh tool. The eighth instrument focuses on gender identification in the text. In the frame of the article four of the eight instruments and specially created computer programs are considered in detail.
\end{abstract}

Keywords - suggestive potential, verbal model, different languages, mechanism of association, computer simulation, analytical tools

\section{INTRODUCTION}

Linguists are often reproached by engineering experts for the absence of the objective methods of data analysis. Those reproaches are presented just in many ways if one speaks about the traditional linguistic approach to the language phenomena analysis which is oriented towards the descriptive model of a language as a stable "solid" product based on the linguistic world-image which is derived from language units using logical-rational analysis of vocabulary units and texts. Such reproaches lose their power as soon as one approaches a human's speech organization as a self-organized system, as a dynamic functioning system, which state is characterized by constant interaction between the process of speech event analysis and its product. Viewing the language as the property of an individual who makes use of it, this approach to the language phenomena analysis allows us to develop algorithms for the analysis of words and texts functioning in human mind, to put forward new teaching techniques, new experimental methods of verbal model analysis in order to overcome communicative challenges, control methods revealing the formation of communicative competence, the procedures of compiling new type of associative dictionaries which are based on the "living knowledge".

In psycholinguistic investigations, experiment and modeling are actively used. As a rule, psycholinguistic work presupposes a great deal of analysis of huge experimental material involving laborious processing mechanisms. The idea of creating computer programs for the automated analysis of the word and the text is not new, but the created programs have not always been destined to survive. Some of them have never been fully applied because of lack of social demand, while the development of others has been cancelled for some reasons.

There are a lot of difficulties and obstacles on the way from understanding the suggestive potential of a verbal model to the creation of special instruments and understanding the laws of word effects. The results of studying the concept of suggestion from the point of view of medicine and psychology made it possible to acknowledge the word as the signal of signals capable of replacing the effects of any physical stimuli. One can only regret the fact that the linguistic achievements in the study of latent verbal mechanisms have not been so significant up to now. There are still some questions as to how to measure the power of word (text) effects, how to determine its qualities and what analysis units can be applied in decoding the suggestibility of language phenomena.

\section{BASIC CONCEPTS}

Let us dwell upon some notions used in the article. The notion verbal model means a model of any complexity (a sound-letter, a word, a text) that carries an inner form which manifests itself in the outer environment thus realizing the latent information capacity and the affecting potential of a particular model. Let us define the suggestive potential as "verbal model force" which can be decoded and measured.

In the effort to measure the suggestive potential of the verbal models (the word/text "power") through studying associative processes, the author tried to single out the analysis units for the levels which make it possible to take measurements and to specify the most advanced directions which help us to create instruments (tools) for detecting the word or text impact in the Russian, English, German, Bashkir, and Tatar languages. 


\section{PSYCHOLINGUISTIC TOOLS}

The first tool deals with of the results of the brain's rhythmic activity which come from reactions to the offered verbal stimuli. The human mind functions within the wide range of the brain's rhythms. Various verbal models (especially the polar ones) are a unique source activating the whole range of man's world perception. The analysis of the brain's rhythmic activity during the perception of different verbal models makes it possible to draw conclusions about their quality and effect. In 2005 an article was published presenting the results obtained by us during the analysis of core units of human mental lexicon through the prism of brain waves emerging during the perception of these units [1]. In recent years a lot of observations and experimental material for consideration have been accrued and are subject to systematization [2]. Brain waves or brain rhythms represent a spontaneous neuron activity which is partially of electric nature. This electric activity can be recorded with the help of electroencephalograph.

High frequency beta-activity of the brain which is characteristic of wakening state structures our perception of the object world, forms the world image and that very "state of alert" in which a human being interacts with the world. This kind of rhythmic activity being very strong suppresses other signals that come in form of alpha and delta-rhythms [3]. There are several types of waves.

1. Beta-waves, which are the fastest, with high amplitude; the wave frequency is in the range of $15-35 \mathrm{~Hz}$. Beta-waves are typical for the active brain engaged in some activity. The beta-state is supposed to be connected with mental or physical tension.

2. Alpha-waves were discovered by the German psychiatrist Hans Berger who while studying the electrical activity of the brain traced weak fluctuations with the frequency of 10 waves per second and he named them alphawaves. The alpha-rhythm is admitted to be the most distinct sample of all the structured manifestations of electrical brain activity. The amplitude of alpha-waves is higher and the frequency is $8-14 \mathrm{~Hz}$. There is a viewpoint according to which human visual perception is connected with alpha-rhythms. The alpha state is a state of peaceful wakefulness, a state of relaxation and meditation.

3. Teta-waves have even greater amplitude with the frequency of 5-8 Hz. The teta-state can be characterized as a state of mental relaxation. Weakened alpha-waves which are typical of the wakefulness state are gradually replaced by tetarhythms. Man feels sleepy. The consciousness control becomes weaker or completely disappears, actions become automatic. Teta-rhythm is also considered to correlate with emotional tension. Sometimes it is called "stress-rhythm" or "the rhythm of tension". One of the symptoms of emotional excitement is the strengthening of the teta-rhythm with the frequency of 4-7 Hz that accompanies both positive and negative emotions [4].

4. Delta-waves with the maximum amplitude and minimal frequency $(0,5-4 \mathrm{~Hz})$ are typical of deep sleep (the one without any dreams and with the predominant frequency of $2-3 \mathrm{~Hz}$ ).

The frequency and amplitude of waves depend on the synchronic cooperation of neuron cells. The long-wave diapason is possible only with the synchronic work of cells for in this case the brain becomes sensitive to weaker signals too. The most consistent example here is the state of meditation during which the inner monologue ceases. And as physicists would say nonlocal superposition state appears. The same state arrives during deep praying; in this case, the electric activity of the brain (low wave delta-rhythms) can serve as an indicator of this state. The analysis of the brain's rhythmic activity during the perception of various verbal models can help us to make a conclusion about the quality and strength of their effects.

The second tool which also seems rather promising is connected with research in the field of associative (psychological) coloring of the sound [5-9]. It is evident that mathematics calculations and computer modeling are impossible without determining the exact and finite amount of analysis units. This condition is extended to all language phenomena and the choice of this or that language material does not matter. The problem consists in language space itself: not all levels of the language system make it possible to single out the finite (and not too large) amount of units for the analysis. The material elements of sound-imagery system of a particular language containing semantic and formal aspects offer simultaneously different units. The author chose soundletters them being units of sound-imagery system first of all because of their finite amount, i.e. it is possible to count all the possible combinations and then to model and program the obtained data, to create automated information systems for further research. Another positive aspect is that one can work almost with any language. There is one more supposition in favor of this unit. For a speaking person a sound becomes real only after corresponding it to a letter. That is why an unknown set of sounds perceived audibly and not supported with a visual letter image will not provide us full understanding because of minimal differences in feature shells. The study of the phonosemantic specificity of idiolexicon core units confirms the fact that the phonetic level of the language participates in the formation of associative bonds and realizes them in a particular way. The sound level being formed first in ontogenesis makes it possible to reach this unconscious level that takes part in associative processes. Modern phonosemantics nowadays has psychophysiological basis that is enough to approach the problem of sounding seriously. The phonetic level is the one at which a person experiences the greatest sensory effects. Synesthesia and kinematics being psychophysiological basis of sound-symbolism and referred to as perception phenomena make it possible to decode information through the appearance of supplementary feelings or images typical of another modality (synesthesia) and through accidental muscle contractions which accompany feelings and emotions (kinematics).

This research work is based on the different languages (Russian, English, German, Bashkir, and Tatar) [10, 11]. Analyzing the associative (psychological) coloring of the sound, let us rest upon the sound-letter meaning a polymodal 
entity perceived by way of several sensory channels (at least audial and visual). Human perception is polymodal. Intermodal interactions result from the united nature of the surrounding world which underlies the deep bonds between different perceptive systems. An audial presentation can cause unconscious appearance of visual images or verbal associations for it already contains the generalized image resulting from the operation of the cognitive mechanism of emotional-evaluative generalization and differentiation. It is also certain that one deals with an associative metaphoric parallel between the sound and the meaning, which is based on emotional-semantic evaluation of the sound that allows us to realize the intermodal traverse "sound-emotion-feature". When one makes use of one of the most favored in psycholinguistics metaphors "living knowledge", let us consider associations as the product of functioning of an individual's activated systems which present a peculiar characteristic of a person as a living system determining the quantity and quality of his interaction with other systems.

The acoustic aspect of speech, acoustic changes are not located in "the highlighted part of the mind" of a language speaker. In order to understand them and for the purposes of comparative studies on the basis of different languages it is necessary to bring them out to the conscious level. The author tried to materialize and present in the form of color matrixes one of the components of the suggestive nature of soundletters, i.e. their associative coloring. For the purpose of the research psycholinguistic experiments on the basis of different languages were conducted and computer programs were created in order to analyze verbal information and to simulate the associative background of the language.

In 2011 a group of authors (the head of the project T. M. Rogozhnikova, the programmer S. A. Voronkov, the postgraduate students N. V. Efimenko, R. V. Yakovleva) has developed the computer program for automated analysis of the word and the text (State Registration Certificate for computer programs № 2011618299 “BARIN”). The main component of the program includes the color matrixes of the English and Russian language sound-letters. The program code is divided into several modules: the interface module, the general text analysis module aimed at processing any written English or Russian text, the module for determination of the color contents dynamics, the module responsible for the formation of a spiral model of the color image of a text, the module for the computer image interpretation of sound-color correlations in a text, the statistics module. All in all the program implements 9 functions.

In 2013, T. M. Rogozhnikova, D. D. Kudashov, G. R. Kochetova, N. V. Efimenko developed the computer program "BATYR", the main component of which includes the color matrixes of the Tatar and Bashkir language soundletters (State Registration Certificate for computer programs No. 2014613238 "BATYR"). The program code is divided into several modules: the interface module, the general text analysis module aimed at processing any written Tatar or Bashkir texts, the module for determination of the color contents dynamics, the process of imagery-computer interpretation (dynamic image), the module for the determination of sound-color correlations in a text, the stating the main colors and the presentation of correlations in the form of a color spiral, the statistics module. All in all, the program implements 12 functions.

In 2016 T. M. Rogozhnikova, D. D. Kudashov, R. V. Yakovleva have developed the computer program "BURGER" the main component of which includes the color matrixes of the German language sound-letters (State Registration Certificate for computer programs № 2016616320 "BURGER”). The program code is also divided into several modules and the program implements 12 functions.

In the picture (Fig.1), there is an illustration: a slice of the process imagery-computer interpretation (dynamic image) of a text (I LOVE YOU) in four languages.

One of the important results of the implemented work is the construction of models presented in a form of pictures displaying associative coloring where the author coded in equal proportion all the sound-letters of the Russian, English, Tatar, Bashkir and German languages. These pictures (Fig. 2) can be taken as a language color background which has absorbed the national-cultural specificity.

The idea to create such models was offered by T. M Rogozhnikova and the technical part was carried out by D. D. Kudashov. The author tried to find a material analogue or the outer form of the material realization of the virtual inner form that is described as associative coloring of linguistic units which "build" the language itself. The associative coloring of the Russian sound-letters is rich in the blue color and its tints. The associative color scale of the English language has orange and yellow coloring providing a bright sunny background. The psychological coloring of the Bashkir language is close to pistachio green, while the Tatar language sound coloring is emerald green. The German language is violet.

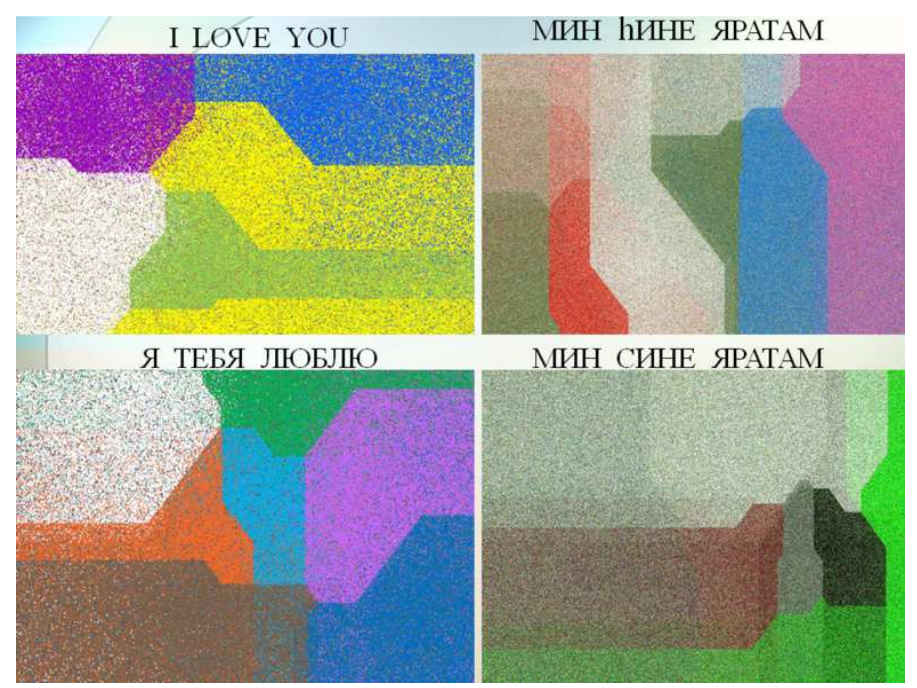

Fig. 1. The associative coloring of a text in Russian, English, Tatar and Bashkir 


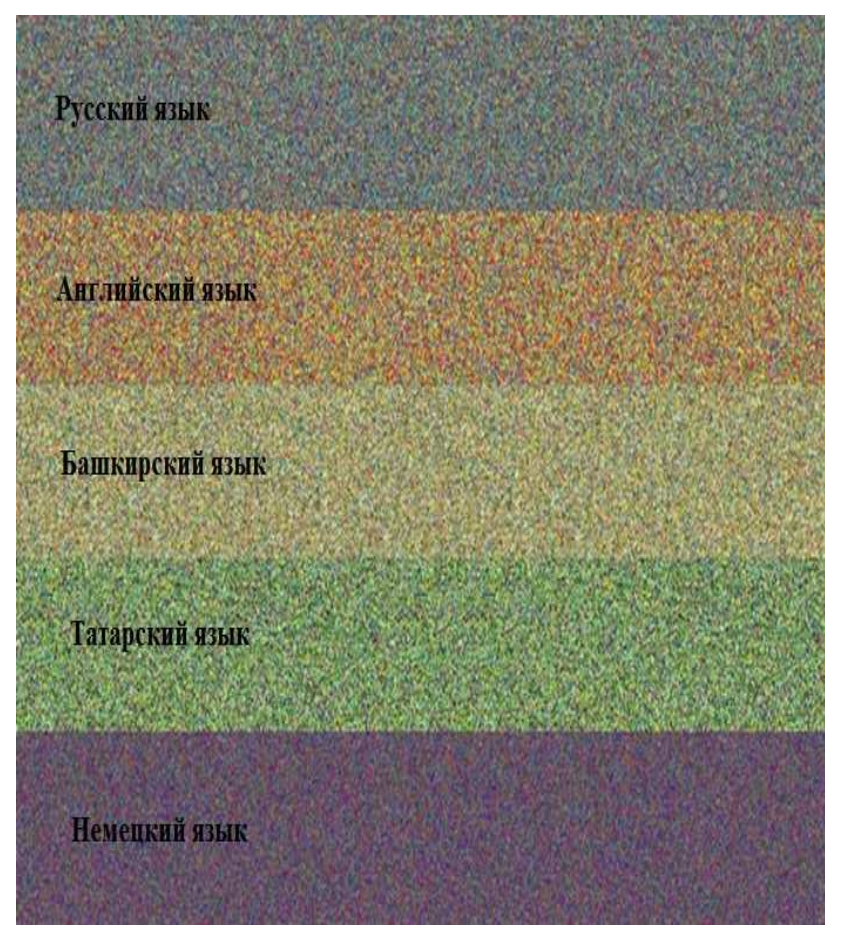

Fig. 2. The associative coloring of the Russian, English, Tatar, Bashkir and German languages (associative color background)

In order to recheck the "accuracy" of the created models, it was necessary to find another way of determination of the associative background of the language. Thus the method of sound-letter frequency determination was chosen: it was carried out through the analysis of a great amount of texts of various genres and the comparison of texts belonging to some particular genre with the already obtained "exemplary" (average) indicators. Gradually the author collected a database which included different discourse types; the author also formed an "exemplary" model based on the sound-letter frequency indicator. It was essential to adhere to the aim that is to create a dynamic verbal modal that could be adjusted if necessary for language is living knowledge developing constantly.

In 2014, the computer program "SCHETOVOD" ("accountant") was written for this purpose. (State Registration Certificate for computer programs №. 2014618598 "SCHETOVOD”). The code of the program is divided into several modules: the text recalculation module, the recording and reading module for the adjacent database, the database interpretation module. By means of this software product, it is possible to calculate the quantity of sound-letters of the analyzed Russian text; to determine the frequency of sound-letters of the analyzed text, and also to compare the obtained results with the average model indicators; to work out the average model of the Russian language basing on analyzed texts; to compare the frequency of sound-letters contained in different topic texts with the average model; to provide information in the form of schedules, charts and texts. The program is intended for universal use. The automated sound-letter analysis in the word or in the text is possible for any types of Russian texts.
The first (experimental) variant of the "exemplary" model was constructed on the basis that includes nearly six million processed sound-letters (fiction texts, scientific, publicistic, advertizing, suggestive (prayer) texts, as well as political discourse). Statistics is available for all text types indicating the quantity and frequency of emergence of sound-letters in all texts and the quantity of the processed sound-letters of each genre. As a result, it was possible to compare the "exemplary" model obtained on the basis of frequency with the model created by the principle of equal "color" share of each language sound without the support on the frequency indicator. The models are almost identical so it is possible to say that these obtained results can be considered as reliable ones.

At present, the associative color background of other studied languages is being rechecked. The author also continues her work on the creation of computer programs similar to "SCHETOVOD", but they are meant for the English, German, Tatar, and Bashkir languages.

The "exemplary" associative coloring can serve as a quite informative source for characterization of the impact that a particular model can have upon a person for any significant "color deviations" will no doubt prove that there are different impact vectors. In order to follow this trend, one of the tasks to be completed will be the creation of a typology of associative color deviations for the average model has already been made and it can serve as a standard. It can be also challenging to analyze psychological comfort a person has while using a foreign language which enters his mind being unfamiliar and unnatural from the point of view of its coloring. The consequences of such "intervention" that introduces a kind of discord or on the contrary makes the language rainbow more harmonious have never been studied as one can judge by the available research.

The third tool. If (according to quantum mechanics) full description of any phenomena is based on "the state vector" that represents a total of mutually exclusive states, then it is possible to choose a particular state vector as a superposition of two mutually exclusive states. The author considered the superposition of two exclusive states - bad / good. The most entangled state in this case is the state "neither good nor bad" - a kind of a neutral zone. One can consider the whole range of states within this basis depending on the amplitude indices of the superposition. It is necessary to remind that the principle of states superposition is described as follows: if a system can reside in different states, it can reside in two (or more) states simultaneously. The quantum superposition is a superposition of alternative (mutually exclusive) states. In this case the description of the psychological structure of word semantics can be carried out within the borders of the finite space of states with particular dimensions. Thus the core units of man's mental lexicon were analyzed. Individual and collective associative fields were studied. Individual semantics is limited by the number of possible states; at that, the development of the semantics becomes real due to the potential that makes it possible to enlarge the space of states. The polarization of world aspects is objective while the unification of one polarity with another one results from man's subjective perception. Human everyday thinking is 
characterized by an amazing "cognitive miserliness", human mind does not burden itself with informative categories, but it is more likely to make use of emotional-evaluative categories. In the process of interaction with the material world (in this case, it is the interaction with a verbal stimulus) that triggers a decoherence process destroying the superposition state bad / good, one can detect the manifestation of the material object (in this case it is a verbal association) that was potential previously and the vector of state becomes either "good" or "bad". The state vector or, to be exact, the deviation of the vector towards one of the options (good / bad) can change. These changes can be registered during the analysis of "the state" of an individual associative field fragment as a product (or a result) of such measurements. The measurement of the state makes it "manifest itself" and singles it out as a separate independent object of reality which is further registered as a basic form, "good" or "bad".

A peculiar "stagnation" of associative reactions that is clearly seen in stereotypical associative clusters which are unlikely to change over a significant period of time can be explained by a decoherence process which, being a physical process, takes place in a human being's mind thus pulling him into narrowed space of perception and bringing these stagnation phenomena in the form of long-lasting associative fixations and associative dependence.

The forth tool is connected with the study of rhythmic codes of verbal models. The author suggested that texts with different rhythmic structures have different suggestive potentials. In an arbitrary way, a text can be divided into high, medium and low rhythmical recurrence texts. This suggestion is based on the specificity of human mind, which according to $\mathrm{W}$. Wundt is rhythmic in its nature. This phenomenon is closely connected with human psychological organization. Human mind is rhythmic because, on the whole, human body is rhythmic [12].

The complexity of rhythm studying is due to its peculiarities for a human being perceives these recurrences of comparable and sensuously feasible units both emotionally and somatically (corporally), but they are scarcely expressed through language phenomena. Researchers are concerned about this specificity while trying to single out minimal rhythmic units of verbal models. Analyzing research papers by national and foreign authors L.V. Kishalova points out that the most common units are syntagms and rhythmic groups. But a number of scholars compile whole hierarchies of rhythmic units taken from prose [13]. So in some research papers, a syllable, tact, a phonetic sentence, an intonation entity are emphasized as rhythmic units. At the same time, other papers single out a colon, a phrase component, a phrase and super phrase construction (paragraph); a phonetic word, a syntagm and a sentence; stressed syllables, syntagms and intonemes and others. G. N. Gumovskaya following A. M. Antipina supposes that the rhythmic structure of prose can be precisely describes through the construction of a tonogram of a prosaic text. Singling out the rhythmic group, syntagm, phrase, super phrase unit as analysis units, the author calculated the rhythmic module as a mathematical characteristic of prose rhythm in English texts belonging to different genres. In accordance with the results, G. N. Gumovskaya made a conclusion that the most rhythmic texts belong to journalistic genre. For the purpose of this research, it is important to mention another peculiar notice proving the existence of an author's rhythmic module (i.e. the rhythmic module of different pieces of work by the same author) that is a constant value. This fact makes it possible to identify the author of a certain text [14].

The problem of rhythm analysis units has not been solved yet that makes it difficult to develop an effective and universal method of the verbal model rhythm determination which would be based on mathematical calculations. In 2015, T. M. Rogozhnikova, L. V. Kishalova, A. E. Kishalov developed a computer algorithm for the analysis of the text rhythmic recurrence level, for the determination of the rhythm stability periods and its measurement borders. The algorithm is based on the minimal basic units of analysis (a syllable, a rhythmic group, a syntagm). The interchange of rhythmic units creates a certain overrhythm and their aggregate makes the rhythmic structure of the text. (Computer program for automated analysis of text "PULS". State Registration Certificate for computer programs № 2015614549 “PULS”).

Through the determination of rhythmic coefficients and their ranging in accordance with the obtained results, the given software would allow us to create "standard" rhythms of verbal models, the suggestive potential of which would give us an opportunity to solve various application tasks including the creation of academic and scientific texts that are easier and more convenient to perceive.

\section{CONCLUSION}

So four of the eight possible tools have been considered. The first tool deals with studying rhythmic activity of the brain while presenting verbal stimuli. The second one relates to the analysis of psychosemantics of color and associative color of models. The third one bases on possible analogies with the models used in quantum physics. The fourth one touches upon the rhythmic organization and rhythmic codes of the models.

The remaining instruments will be covered in another article. The fifth direction is oriented to the analysis of language phenomena in the context of different perception modalities. The sixth one focuses on ranking emotionalevaluative features characteristic of the verbal model. Suggestive resources of musical discourse are analyzed in the context of the seventh direction. The eighth direction focuses on gender identification in the text.

Modern linguistics and psycholinguistics in their complexity can easily compete with mathematics and physics. Experiment and simulation as psycholinguistic tools for verbal models analysis are useful in improving discourse color climate, and computer technologies make it possible to determine the quality of already existing models, predict their suggestive potential and create verbal membranes with the predetermined characteristics, which code information about the surrounding events into ecological models of language communication. 


\section{References}

[1] T. M. Rogozhnikova, "The Brain Waves and the Core of the Mental Lexicon," in the collection of articles "The Word and the Text: Psycholinguistic Approach”. Tver' State University, 2005, Iss. 5, pp. 96103 (in Russian).

[2] T. M. Rogozhnikova,"Verbal Models and the rhythmic activity of the brain," in The Questions of Psycholinguistics, the edition devoted to E.F. Tarasov's 75th anniversary, Moscow, IYa RAN, No. 2(12), pp. 4856, 2010. (in Russian).

[3] P. M. Walles, Brain Waves, The Psychological Encyclopedia, 2nd edition, edited by R. Korsini, A. Auerbach, St. Petersburg, Piter publishing, 2003, pp. 414-415.

[4] I. V Ravich-Shcherbo, et al. Psychogenetics, Moscow: Aspect-Press , 2004, 447 p. (in Russian).

[5] N. V. Efimenko, Associative structure of word and text color meaning: dissertation can. sc. (philology), Ufa, 2011, 208 p. (in Russian).

[6] L. P. Prokofyeva Sound-color associativity: universal, national, individual. Saratov: Saratov Medical University Press, 2007, 280 p. (in Russian).

[7] T. M. Rogozhnikova, Ethnocultural specifics of color associativity of Bashkir and Tatar sounds, Russkoyazychie $\mathrm{i}$ Bi(poli)linguism in intercultural communication of XXI century: cognitive and conceptual aspects: IV International methodological conference materials. Pyatigorsk: Pyatigorsk State Linguistic University Press, 2011, pp. 51-55 (in Russian).

[8] T. M. Rogozhnikova, and G. R. Kochetova, "Analysis of Bashkir and Tatar sounds' color associativity," Linguistic existence of people and ethnos: psycholinguistic and cognitive aspects: materials of the seminar school (VII Berezinskie chteniya), Iss. 17. M.: the Institute of Scientific Information for Social Sciences of the Russian Academy of Sciences, the Academy of Social Administration, 2011, pp. 241-246.

[9] R. V. Yakovleva, Associative coloring of German sound-letters, The Theory and Practice of Linguistic Communication: VI International Scientific and Methodological Conference Materials, Ufa, UGATU Press, 2014, pp. 365-374. (in Russian).

[10] T. M. Rogozhnikova, et al, Associative dictionary of the Bashkir and Tatar languages, Moscow, Flinta: Nauka, 2016, 364 p. (in Russian).

[11] T. M. Rogozhnikova, et.al., Gallery of associative portraits: Monograph, Ufa, UGATU Press, 2009, 448 p. (in Russian).

[12] W. Wundt "Awareness and Attention", Anthology on Attention. Moscow, Moscow State University Press, 1976, 385 p.

[13] L.V. Kishalova, "The Units of Rhythmic Analysis in Prose," The Theory and Practice of Linguistic Communication: V International Scientific and Methodological Conference Materials, edited by T.M. Rogozhnikova, Ufa, UGATU Press, 2013, pp. 227-232. (in Russian).

[14] G. N. Gumovskaya, "The Rhythm of Prose: problems, search, solutions," Collection of Scientific Papers, Issue 11, Moscow, Institute for the Humanities and IT Press, 2008, pp. 4-37. (in Russian). 